\title{
Audio Codecs Impact on Quality of VoIP Based on IEEE802.16e Considering Mobile IP Handover
}

\author{
Ali M. Alsahlany, Hayder S. Rashid \\ Department of Communication Engineering, Engineering Technical College / Najaf, Al-Furat Al-Awsat Technical University, Najaf, Iraq \\ Email address: \\ alialsahlany@yahoo.com (A. M. Alsahlany),hyder_hejajoo@yahoo.com (H. S. Rashid) \\ To cite this article: \\ Ali M. Alsahlany, Hayder S. Rashid. Audio Codecs Impact on Quality of VoIP Based on IEEE802.16e Considering Mobile IP Handover. \\ American Journal of Networks and Communications. Vol. 4, No. 3, 2015, pp. 59-66. doi: 10.11648/j.ajnc.20150403.17
}

\begin{abstract}
A simulation model using OPNET tool is introduced for testing audio codecs impact on quality of VoIP based on IEEE802.16e with taking into account handover performance. Different parameters that indicate the quality of VoIP such as Throughput, MOS, End to End delay and traffic send and received. According to our simulation the acceptable MOS value is recorded for codecs G.711 and GSM-FR.
\end{abstract}

Keywords: Audio, Codecs, QoS, WiMAX, Handover

\section{Introduction}

In the recent years the Worldwide Interoperability for Microwave Access (WiMAX) placement is growing rapidly. IEEE 802.16e (Mobile WiMAX) becomes a prevalent technology for holding mobile clients because the base station (BS) of mobile WiMAX has the vital benefit to serve considerable coverage areas. During data transmission, mobile WiMAX permits the user to move freely. The major consideration of mobile WiMAX is that when the user is moving from one BS to another there must be no loss in data, i.e. during the handover which represents the method when a mobile node $(\mathrm{MN})$ alters the serving BS [1]. Serving a large number of MNs practically demands an effective scheme of handover. Presently, a considerable delay in handover of mobile WiMAX is contributed to the overall end-to-end communication [2]. In the recent researches more attention is paid to increase the handover schemes efficiency. In this research, a comparison to study the performance of Mobile WiMAX handover among various audio codec schemes will be examined. The rest of this research will be structured as follows: the next section will object to the related works. Section 3 refers to the WiMAX handover procedure. In section 4, voice encoding schemes of utilizing VoIP over IEEE802.16e network will be clarified. Section 5 presents network model implementation. In Section 6 the simulation results will be discussed and finally, conclusion will be stated in section 7 .

\section{Related Works}

Our study recognizes a number of earlier researches in the same course. The main contribution in [3] is to assess and examine the performance of several audio encoding schemes employing RSVP in a VoIP based on WLAN. The results were compared among three audio codecs namely; G.711, G.723.1 and G.729 utilizing the services of RSVP. On the other hand, the researchers in [4] examined mobile WiMAX performance using voice codecs under only two conditions and showed that the performance of audio codecs stay fixed for random way point and group mobility manner.

The researchers in [5] introduced an investigation to study the performance of different audio codecs based on various service classes such as rtPS, ertPS, nrtPS, Best Effort and NOAH routing protocols. While the authors in [6] introduced the performance evaluation of VoIP in wireless networks considering packet end-to-end delay and throughput without taking into account the mobility of nodes. In [7] the study examined the handover in order to delay characteristic and throughput involving UDP and TCP protocols for two types of fading models. An efficient technique of forced handover to progress capacity handling and performance of base station in [8] was introduced; however, the study did not mention audio performance during handover. In [9] the researchers provided an evaluation for different scenarios of WiMAX handover with several movements. While in [10] the authors presented an examination of handover impact on the speech performance in VoIP but audio codecs did not take 
into consideration. This study will provide an examination of the performance of WiMAX handover using four kinds of audio codecs namely; G.711, G.723.1, G.729A and GSM-FR.

\section{IEEE 802.16e Handover Procedure}

In general, due to weak signal received, cell capacity and radio channel condition the handover is occurring. The most important component before occurring handover process is the received signal strength. The $\mathrm{MN}$ will exchange to the adjacent BS if a significant drop in the received signal strength is observed $[7,8]$. The procedure of handover can be made according to the following phases.

- Phase 1: Cell Reselection

Reselection of cell indicates to the procedure that $\mathrm{MN}$ is scanning and/or associating with one or more BSs according to fix handover suitability taking into account other performance issues. The MN might be used the acquired data of neighbor BS from a decoded data to arrange intervals of scanning or sleep intervals to scan the neighbor BS for the reason of evaluating the handover of $\mathrm{MN}$ to a potential target BS $[8,9]$.

- Phase 2: Handover Decision and Initiation

The decision to transfer the mobile station to the desired BS from the serving BS is known as initiation of a handover. Both BS and MN can be started this decision. Starting the actual handover requires sending a handover request that will activate a specific messages sequence of handover to be sent between $\mathrm{MN}$ and BS $[8,9]$.

- Phase 3: Synchronization

The synchronization of MN to its downlink channel is required to create the communication with the desired BS. In this stage, the MN starts receiving the uplink and downlink transmission parameters. This process can be reduced if the $\mathrm{MN}$ earlier received data about the desired BS (during the acquisition of network topology) [8,9].

- Phase 4: Ranging

The target BS and the MN must have handover ranging. Re-entry of network progress from the initial ranging steps in the network entry process negotiates basic capabilities, authentication stage, establishment stage, registration and optional network entry steps. Network re-entry may be reduced by target $\mathrm{BS}$ possession of information of $\mathrm{MN}$, acquired from serving BS over the backbone network. Based on the amount of the acquired information, the target BS may choose to avoid one or several of network entry steps [8]. Regardless whether the serving BS received MN information or not the target $\mathrm{BS}$ will demand $\mathrm{MN}$ information from the backbone network $[8,9]$.

- Phase 5: Termination of Mobile Node

Termination of the MN context is expressed as the serving BS termination of the context of all connections belonging to the $\mathrm{MN}$ and the removal of the context associated with them $[8,9]$.

\section{Voice Encoding Schemes}

Voice codecs are used to convert an analog voice signal into digitally encoded version. The codecs may vary in sound quality, required bandwidth and computational requirements, etc. Table 1 shows the commonly used voice codecs with their algorithms, bit-rates and mean opinion score (MOS) [4]. Voice codec can be briefly defined as an algorithm used to code and decode voice stream. The voice quality experienced by the end-user depends on the performance of the codec [11].

Table 1. Features of the most common codecs.

\begin{tabular}{|c|c|c|c|c|c|c|}
\hline IUT-T Codec & Algorithm & Frame (ms) & Bit Rate (kbps) & Bits per frame & Codec delay (ms) & Comments \\
\hline G.711 & $\begin{array}{l}\text { Pulse code modulation } \\
\text { (PCM) }\end{array}$ & 0.125 & 64 & 8 & 0.25 & $\begin{array}{l}\text { Delivers precise speech } \\
\text { transmission. Very low } \\
\text { processor requirements. }\end{array}$ \\
\hline G.723.1 & $\begin{array}{l}\text { Algebraic Code Excited } \\
\text { Linear Prediction (ACELP) }\end{array}$ & 30 & 5.3 & 159 & 67.5 & $\begin{array}{l}\text { High compression with } \\
\text { high quality audio. Can use } \\
\text { with dial-up. Lot of } \\
\text { processor power. }\end{array}$ \\
\hline G.729A & $\begin{array}{l}\text { Conjugate Structure } \\
\text { Algebraic Code Excited } \\
\text { Linear Prediction } \\
\text { (CS_ACELP) }\end{array}$ & 10 & 8 & 80 & 25 & $\begin{array}{l}\text { Excellent bandwidth } \\
\text { utilization. Error tolerant. }\end{array}$ \\
\hline GSM FR & $\begin{array}{l}\text { Regular Pulse Excitation } \\
\text { Long Term Predictor (RPE- } \\
\text { LTP) }\end{array}$ & 20 & 13 & 260 & 40 & $\begin{array}{l}\text { High compression ratio. } \\
\text { Free and available in many } \\
\text { hardware and software } \\
\text { platforms. Same encoding } \\
\text { is used in GSM cell phones }\end{array}$ \\
\hline
\end{tabular}

\section{Network Model Description}

The configuration of the suggested Mobile WiMAX network model will be done using OPNET environment. OPNET simulator is chosen to its global recognition in terms of accuracy and reliability to get the results which are much closed to the real life. Also, it provides the necessary modules to study the performance of Mobile WiMAX (IEEE802.16e) with respect to the impact of audio codecs, mobile IP, handover and other mobility features.

The suggested model will be implemented using four 
different scenarios to evaluate the best voice encoding schemes of utilizing VoIP over IEEE802.16e network. It is taken into account the impact of mobile IP and a handover mechanism which occur during the movement of $\mathrm{MN}$ across the cells. In the proposed simulation, all scenarios have the same structure and topology and the comparisons will be conducted using different values of parameters.

The first scenario will implement with voice encoding
G.711 over mobile VoIP handover environment. The second scenario will employ with G.729A audio codec over mobile VoIP handover environment, while the third scenario will be examined using G.723.1 and finally, GSM audio codec over mobile VoIP handover environment will be used in scenario four. The network topology of the simulation environment is illustrated in Figure 1.

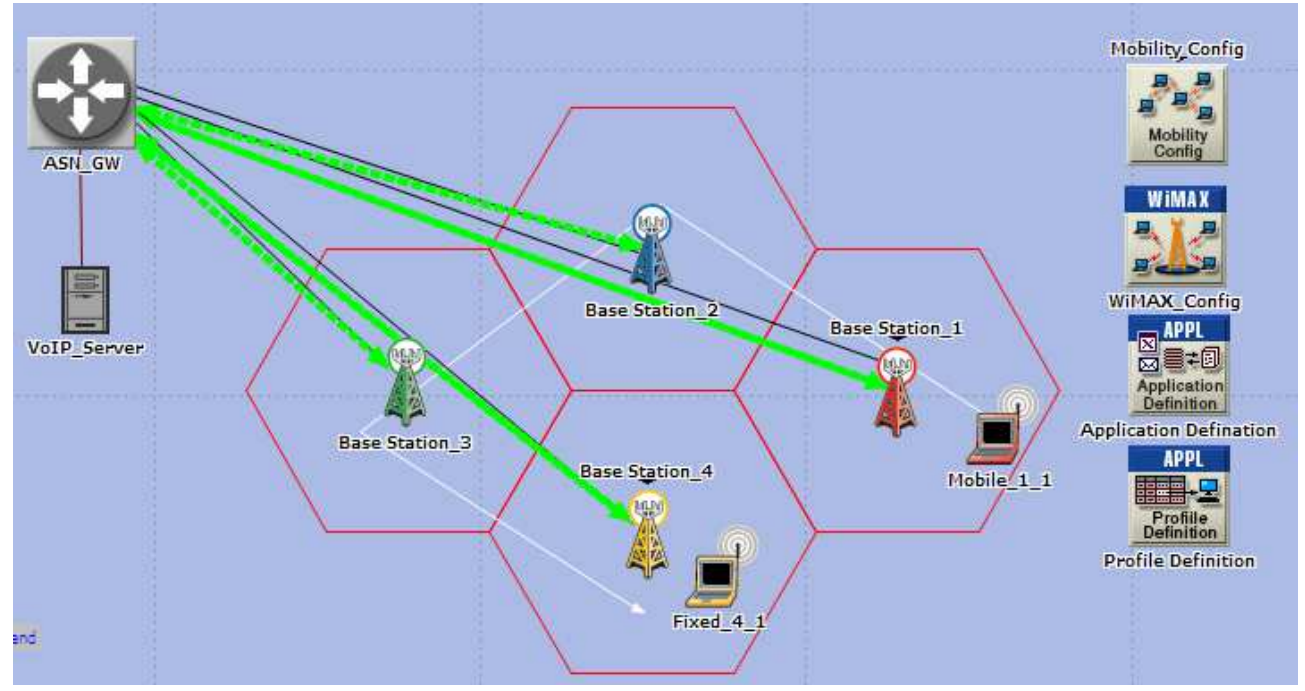

Figure 1. Network Topology of IEEE802.16e.

The scenario of the simulated network consists of four hexagonal cells of IEEE802.16e networks, i.e. single BS per cell. All BSs are connected to the core network (VoIP Server) via Access Service Network Gateway (ASN-GW). The ASNGW supports the mobility in IEEE802.16e network and the VoIP_Server handles the VoIP traffic. The researchers use two MNs with varying movements. The first one is (fixed_4_1) fixed and another node is (mobile_1_1) moved along with the trajectories indicated by a white color line around the cells. The green bidirectional dotted lines represent the generic routing encapsulation (GRE) tunnels. Each BS connected to the ASN-GW by using the PPP DS link which has a $1.544 \mathrm{Mbps}$ capacity. The name of the link in OPNET model is 'point to point_link_adv'. The ASNGW is connected to the VoIP_Server by using 1000BaseT Ethernet cable which has $1 \mathrm{Gbps}$ capacity.

The names of the ASN-GW and servers in OPNET model are 'ethernet4_slip8_gtwy' and 'ethernet_server', respectively. The VoIP node model is 'wimax_ss_wkstn_adv' and the BS (Foreign and Home agent) model is 'wimax bs router adv'. The WiMAX parameters of MNs and BSs are given in Table (2) and Table (3) respectively.

Table 2. Mobile Node Configuration Parameters.

\begin{tabular}{ll}
\hline Application & VoIP \\
\hline Antenna Gain & $-1 \mathrm{dBi}$ \\
Max Power Transfer & $0.5 \mathrm{~W}$ \\
PHY Profile & Wireless OFDMA 20 MHz \\
Modulation and coding & Adaptive \\
Buffer Size & $64 \mathrm{~KB}$ \\
Number of Transmitter & SISO \\
\hline
\end{tabular}

Table 3. Base Station Configuration Parameters.

\begin{tabular}{ll}
\hline Antenna Gain & $\mathbf{1 5} \mathbf{~ d B i}$ \\
\hline Max Power Transfer & $0.5 \mathrm{~W}$ \\
PHY Profile & Wireless OFDMA 20 MHz \\
Modulation and coding & Adaptive \\
Number of Transmitter & SISO \\
\hline
\end{tabular}

\section{- Mobility Configurations}

In our simulation model that shown in Figure (1) we assume that $\mathrm{MN}$ is carrying voice traffic which is ongoing between Mobile1_1 and VoIP_Server. When the simulation time is started, Mobile1 1 initiates the first session with Base station_1 (Home agent). After $9.18 \mathrm{~min}$, the MN performs a handover from the current Home_agent spot where the session was generated to a Base station_2 (Foreign_agent_2) spot. This session will be continued until reaching Base Station_4 (Foreign_agent_4) which is passed through Base Station_3 (Foreign_agent_3). During this movement, the MN will change its Home agent and access address which imply that its IP address also will be changed. According to the handover process, the session at the Home agent will terminate, and the MN has to trigger the handover procedure. The new access router and the foreign BS have not information about the terminated session, so that $\mathrm{MN}$ has to register with the foreign $\mathrm{BS}$ and to establish IP connectivity with new access address and also reestablish session flow with VoIP_Server. Figure (2) shows an IP configuration of our simulation. 


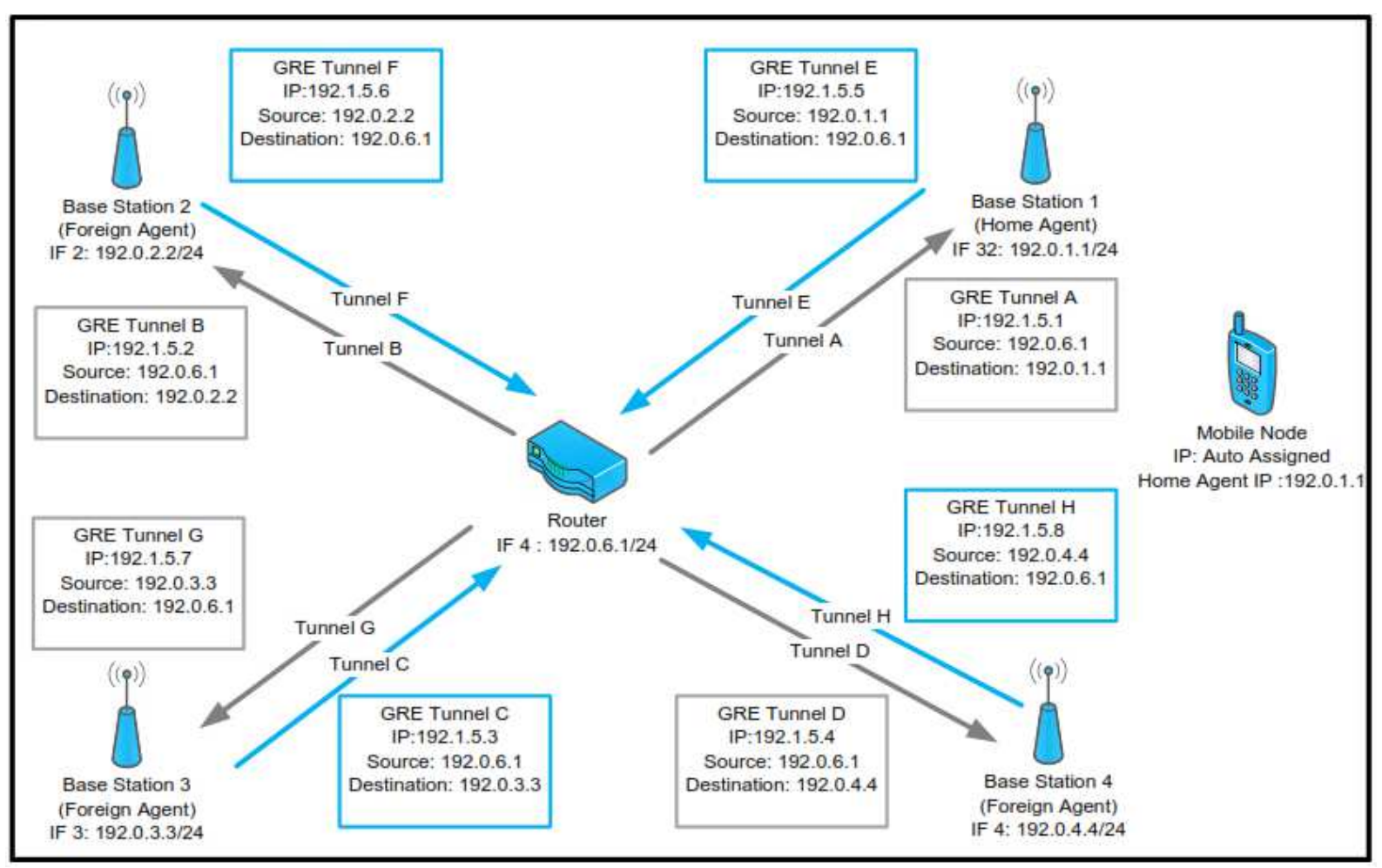

Figure 2. IP configuration for the proposed scenario.

The mobility Configuration defines a mobility profile for the wireless MNs in IEEE802.16e network. The mobility parameters of the simulation scenarios are given in Table (4) and Table (5) respectively.

Table 4. Handover Parameters.

\begin{tabular}{ll}
\hline MS Handover Retransmission Timer (ms) & $\mathbf{3 0}$ \\
\hline Maximum Handover Request Retransmissions & 6 \\
Handover Threshold Hysteresis (dB) & 0.4 \\
Multi target Handover Threshold Hysterias (dB) & 0 \\
Maximum Handover Attempts per BS & 3 \\
\hline
\end{tabular}

Table 5. Scanning Parameters.

\begin{tabular}{ll}
\hline Scanning Threshold (dB) & $\mathbf{3 5}$ \\
\hline Scan Duration (N) (Frames) & 3 \\
Interleaving Interval (P) (Frames) & 255 \\
Scan Iteration (T) & 5 \\
Maximum Scan Request Retransmissions & 8 \\
Ground Speed km/h & 10 \\
\hline
\end{tabular}

The application Definitions define traffic generation on the network. The VoIP application is set up as the application of the whole network.

\section{Results of the Simulation}

In this section, the results are obtained after implementing the IEEE802.16e network simulation and the statistics are collected by using OPNET Modeler under four different scenarios. The collected statistics from the simulation include Throughput (packet/sec), Mean Opinion Score (MOS), Voice packet end to end delay (sec), Voice traffic send and received (packet/sec). The results of the simulation potentially indicated to the degree of performance of the audio codecs over mobile IP handover environment based on voice communication.

The curves in Figure (3) correspond to the throughput of Mobile_1_1 node versus simulation time with the four audio codecs: G.711, G.723.1, G.729A and GSM-FR. According to the gained results, it is observed that the maximum rate of throughput is recorded for the codec G.929A with the rate of 195 packets /sec. On the other hand, G.711 and GSM-FR codecs have the lower throughput rates. The throughput in G.711 codec is ranged from 120 packets/sec to approximately 10 packets/sec, while GSM-FR codec is ranged from 20 packets/sec to roughly 65 packets $/ \mathrm{sec}$. The lowest rate belongs to codec G.723.1 which is oscillated from 5 packets/sec to about 23 packets/sec.

Additionally, it can be clearly seen that throughput of mobile1_1 node is reduced momentarily after $540 \mathrm{sec}, 1320$ sec and $2100 \mathrm{sec}$ respectively; because when the $\mathrm{MN}$ moves outside the home agent coverage area and passes through boarder of foreign agent cell 1,2 and 3 handover will occur. This reduction will continue until handover is completed. During the handover, the Mobile_1_1 node will receive signals from two base stations. When the setup of the new connection is completed, the transmission from/to the serving (Home Agent) BS will be ended, i.e. when the MN crosses a boarder of cells between the serving (Home Agent) BS and target (Foreign Agent) BS, the connection with the serving (Home Agent) BS will be finished. After that, a new connection with the target (Foreign Agent) BS will be 
established.

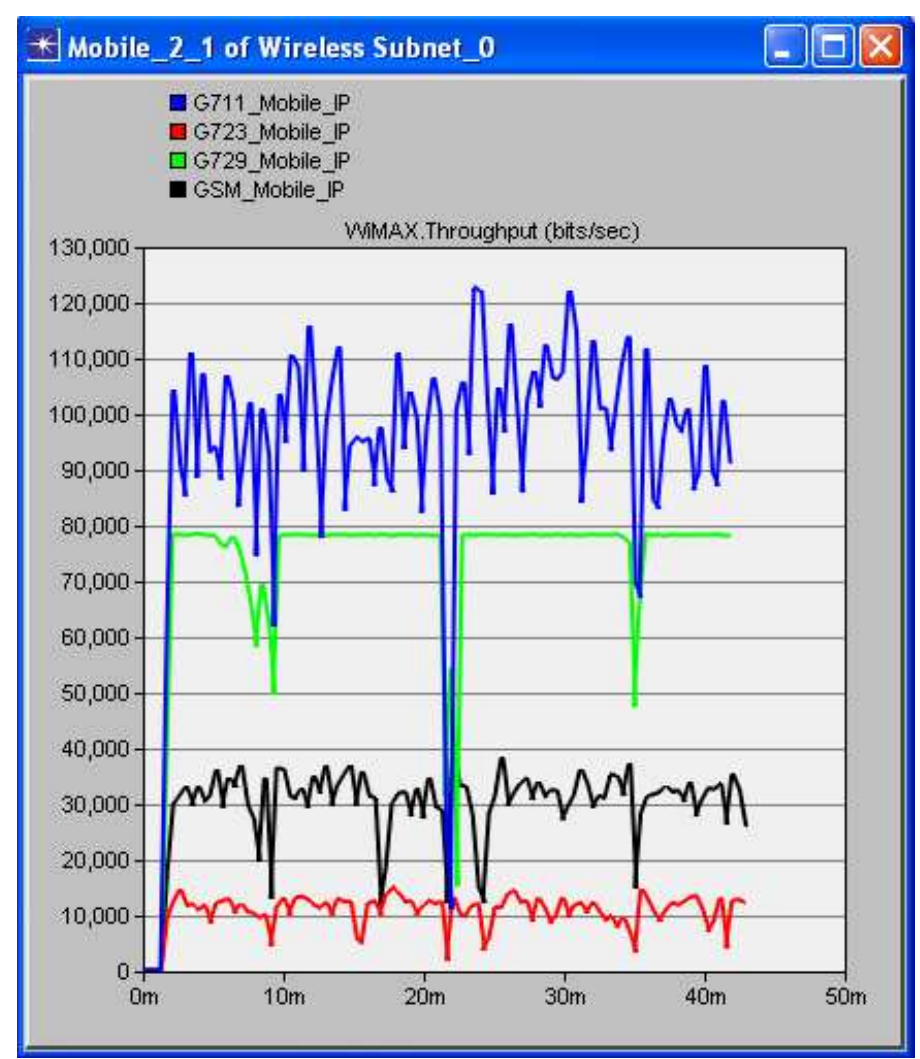

Figure 3. Throughput of various audio codecs.

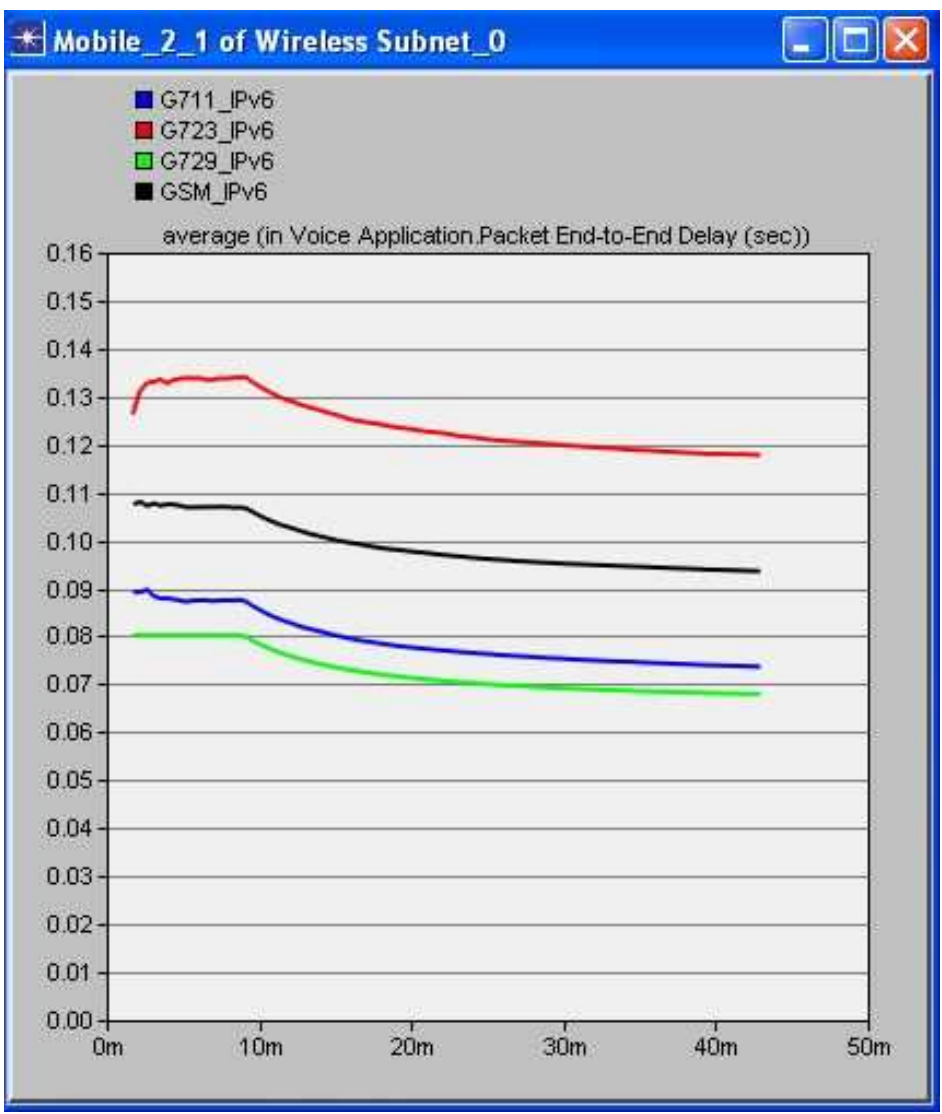

Figure 4. packet End to End delay (sec) under various audio codecs. 
Figure (4) illustrates the obtained results between average end to end delay metric and simulation time. It can be seen that the average end-to-end delays of G.723.1 are larger than $0.11 \mathrm{sec}$ which give a lower performance with respect to the other codecs. This result is due to small transfer rate 5.3 Kbps for G.723.1. On the other hand, the average end-to-end delays of GSM-FR and G.711 are larger than 0.09 second and
0.075 second, respectively, the reasons for that are the high bits per frame which are required more time to process them. While, G.729A has small delay with only $0.08 \mathrm{sec}$ due to the high transfer rate and low packet size. These reasons make the G.729A provide the best performance of VoIP applications over IEEE802.16e network.

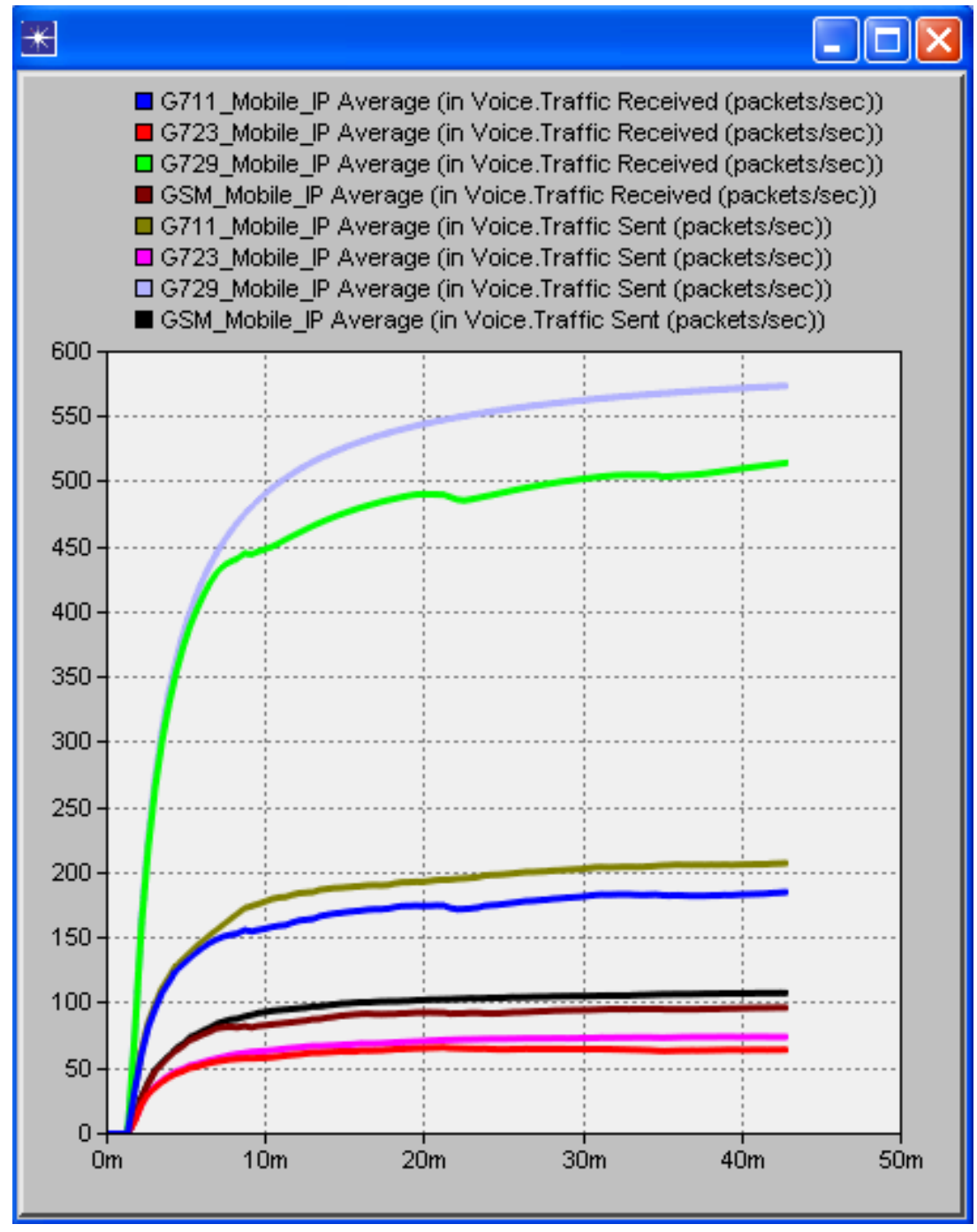

Figure 5. Traffic send and received.

Figure (5) represents the relationship between traffic received and traffic sent versus simulation time with different audio codecs. In order to examine network efficiency, the loss between two traffics (sent and received) must be less enough to make them identical to each other [13]. During the simulation time, the deviation of the traffic received to the traffic sent in G.711 and 729.A codecs is large comparing with GSM-FR and G723.1 codecs that have small deviation. The results indicate that codecs GSM-FR and G723.1 in IEEE802.16e network provide better efficiency since the path loss between traffic sent and received is less compared with other codecs.

Figure (6) shows the average MOS value for the four codecs. The MOS value gives a description of the perceived quality of receiving voice after being transmitted and compressed using codecs. According to [14], the acceptable MOS value in our results is recorded for codecs G.711 and GSM-FR with a value of 3.1 and 3 respectively. In the contrast, the MOS value of codecs G.723.1 and G.729A is less than 3 which mean that these values indicate that the quality of service is poor and cannot be acceptable if these codecs are used. 


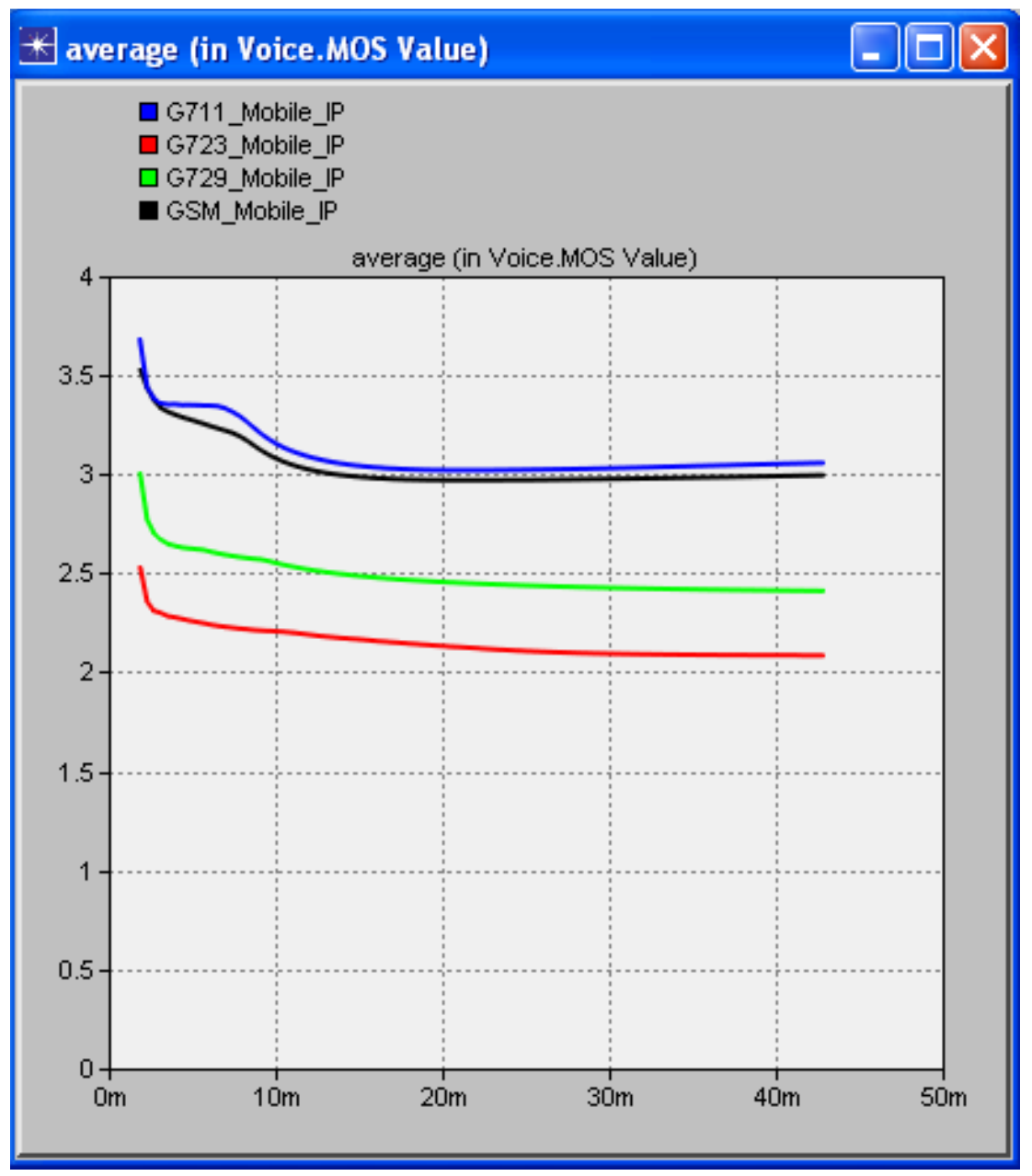

Figure 6. Average voice MOS under various codecs.

\section{Conclusion}

In this study, OPNET Modeler was occupied as a simulation tool for testing audio codecs impact on quality of VoIP based on IEEE802.16e. The proposed handover for IEEE802.16e was validated in various simulation scenarios using four audio codecs. The obtained results show that the acceptable MOS value was recorded for audio codecs G.711 with a value of 3.1 , followed by GSM-FR codecs with a value of 3. Audio codecs G.723.1 and GSM-FR recorded lower effect on handover, i.e. the best performance. Additionally, in traffics sent and received, they have small deviation. In terms of end to end delay, G.729A codec recorded a small delay comparing with other audio codecs.

\section{References}

[1] Rambir Joon, Sandeep and Manveen Singh Chadha, "Analysis of WIMAX Handover", International Journal of Soft Computing and Engineering (IJSCE), pp. 476-479, Volume 2, Issue 3, July, 2012.

[2] Bhaskar Ashoka, David Eyers, and Zhiyi Huang, 'Handover Delay in Mobile WiMAX: A Simulation Study", 12th IEEE
International conference on Parallel and Distributed Computing, Applications and Technologies (PDCAT), pp 305 $-312,2011$.

[3] Priyanka Luthra, and Manju Sharma, "'Performance Evaluation of Audio Codecs using VoIP Traffic in Wireless LAN using RSVP', International Journal of Computer Applications, pp. 15-21, Vol. 40, no. 7, February, 2012.

[4] M. Tariq, M. Azad, and S. Rizvi, 'Effect of Mobility Patterns on VoIP QoS in Mobile WiMAX", International Journal of Computer Science and Telecommunications, pp. 1-7, Volume 4, Issue 1, January, 2013.

[5] Tarik ANOUARI, and Abdelkrim HAOI0, 'Comparative study and analysis of VoIP traffic over WiMAX using different service classes", IEEE International conference on Next Generation Networks and Services (NGNS), pp. 87-93, 2-4 Dec., 2012.

[6] Khamis AlAlawi, and Hussain Al-Aqrabi, ''Quality of service evaluation of VoIP over wireless networks", 8th IEEE conference on GCC Conference and Exhibition (GCCCE), pp. 1-6, 1-4 Feb., 2015.

[7] Mohd Pardi, Mohd Baba, and Muhammad Ibrahim, 'Analysis of handover performance in mobile WiMAX networks", IEEE conference on Control and System Graduate Research Colloquium (ICSGRC), pp. 143 - 149, 2728 June, 2011. 
[8] Shahab Hussain, and et al, "Handoff in mobile WiMAX: Forced handoff scheme with load balancing in mobile WiMAX networks", 8th IEEE International Workshop on Performance and Management of Wireless and Mobile Networks, pp. $666-672,22-25$ Oct, 2012.

[9] Firas Al-Saedi, and Wafa A. Maddallah, 'Evaluation of Handover Process in WIMAX Networks", International Journal of Computer Science Engineering and Technology (IJCSET), pp. 831-838, Vol 2, Issue 1, January, 2012.

[10] Zdenek Becvar, Pavel Mach, and Robert Bestak, 'Impact of Handover on VoIP Speech Quality in WiMAX Networks"', 8th International Conference on Networks, pp. $281-286,1-6$ March, 2009.

[11] S.Nithya, and M.S.K.Manikandan, 'Perform ace Analysis Of CODEC's With QoS Constrainsts In Voice Over Internet
Protocol V6", IEEE International Conference on Electronics and Communication Systems (ICECS), pp. 1-5, 13-14 Feb., 2014.

[12] Jamil Hamodi, and et al, 'Performance Study of Mobile TV over Mobile WiMAX Considering Different Modulation and Coding Techniques", International Journal of Communications, Network and System Sciences, pp. 10-21, Vol.7, No.1, 2014.

[13] Ali M. Alsahlany, 'Performance Analysis of VOIP Trafic over Integrating Wireless LAN and WAN Using Different CODECS", International Journal of Wireless \& Mobile Networks (IJWMN), Vol. 6, No. 3, June, 2014.

[14] Shaffatul Islam, Mamunur Rashid, and Mohammed Tarique, 'Performance Analysis of WiMax/WiFi System under Different Codecs", International Journal of Computer Applications, Vol. 18, No.6, March, 2011. 